\title{
THE TOTAL CURVATURE OF A KNOTTED TORUS
}

\author{
NICOLAAS H. KUIPER \& WILLIAM H. MEEKS III
}

Dedicated to James Eells, Jr. on his anniversary

\section{Introduction}

The classical total curvature of a simple closed smooth $\left(C^{\infty}\right)$-curve $\gamma$ in euclidean three-space $\mathbb{R}^{3}$ is

$$
K(\gamma)=\int|\rho| d s
$$

where $s$ is arclength and $\rho$ is the curvature density. Denoting by $[\gamma]$ the isotopy class of the knot, define the classical total curvature of the isotopy type of $\gamma$ as the greatest lower bound

$$
K[\gamma]=\underset{\gamma^{\prime} \in[\gamma]}{\text { g.l.b. } K\left(\gamma^{\prime}\right)}
$$

Call the $z$-coordinate of a system of euclidean coordinates $(x, y, z)$ the height. Then consider the number of critical points at relative maximal heights on any $\gamma^{\prime} \in[\gamma]$ for which $z \mid \gamma^{\prime}$ is nondegenerate. The minimum of these numbers for $\gamma^{\prime}$ isotopic to $\gamma$ is called the bridge index $B[\gamma]$ of $\gamma$. Fox [4] gave lower bounds:

$$
B[\gamma] \geqslant 1+\sigma_{1}(\gamma) \geqslant 1+\lambda(\gamma),
$$

where $1+\sigma_{1}(\gamma)$ is the minimal number of generators of the fundamental group of the complement $\mathbb{R}^{3} \backslash \gamma$, and $\lambda(\gamma)$ is a number defined in Fox's differential calculus for knots and can be computed for any knot [1]. The invariants $\lambda(\gamma)$ and $B[\gamma]-1$ (see [13]) are additive for connected sums of knots.

There exist knots for which $B(\gamma)>1+\sigma_{1}(\gamma)$, namely the torus knots $\gamma_{p, q}$, $p>q \geqslant 3$, with $B(\gamma)=q>1+\sigma_{1}=2$ [14], and knots for which $\sigma_{1} \geqslant 1>0$ $=\lambda($ see $[13])$. 
The combined efforts of Fary-Fenchel-Fox-Milnor [2], [3], [4], [10], [11] lead to

Theorem 1. The classical total (absolute) curvature of a knot $\gamma$ is

$$
K(\gamma)=\int|\rho| d s \geqslant K[\gamma]=2 \pi \cdot B[\gamma] \geqslant 2 \pi
$$

For a nontrivial knot equality is never attained:

$$
K(\gamma)>2 \pi \cdot B(\gamma) .
$$

Equality, only possible for the unknot $K(\gamma)=2 \pi$, occurs only for plane convex curves.

The classical total (absolute) curvature $K(M)=\int\left|K_{p} d \sigma_{p}\right|$ of an embedded closed surface $M$ in three-dimensional euclidean space is the integral of the absolute value of the Gaussian curvature on the surface, and has the simple equivalent definition as the area counted with multiplicity of the image in the unit sphere of the associated Gauss (or normal) map.

Let $f: T \subset \mathbb{R}^{3}$ be an embedding of a torus $T$ in euclidean three-space $\mathbb{R}^{3}$. We compactify $\mathbb{R}^{3}$ by one point $\infty$ to obtain a 3-sphere $S^{3}=\mathbb{R}^{3} \cup \infty$. If each of the two components of the complement $S^{3} \backslash T$ is standard, that is, diffeomorphic to a regular neighborhood of a circle in $\mathbb{R}^{3}$, then $T$ is unknotted; it is isotopic to a geometrically standard round torus. Otherwise, when $T$ is knotted, exactly one of the two components $T^{u}$ is standard. We will say that a knot $\gamma$ in $\mathbb{R}^{3}$ is a core curve for the torus $T$ if it lies in this standard component, $\gamma \subset T^{u} \subset S^{3}$, and there is a diffeomorphism

$$
\eta:\left(S^{1} \times D, S^{1} \times \partial D, S^{1} \times 0\right) \rightarrow\left(\overline{T^{u}}, T, \gamma\right) \subset S^{3},
$$

where $D$ is the unit disc with boundary $\partial D$ in $\mathbb{R}^{2}, S^{1}$ is the unit circle, and $\overline{T^{u}}=T^{u} \cup T$ is the closure of $T^{u}$ in $S^{3}$. There are many core curves for $T$, but any two are ambiently isotopic in $T^{u}$.

Let $K[T]$ be the greatest lower bound of $K\left(T^{\prime}\right)$ for all $T^{\prime}$ isotopic in $\mathbb{R}^{3}$ to $T$. It is called the classical total curvature of the isotopy type [T] of $T$. We will prove our

Theorem 2 (The generalized Fary-Fenchel-Fox-Milnor theorem). The classical total absolute curvature of a torus $T$ embedded in euclidean three-space $\mathbb{R}^{3}$ obeys:

$$
K(T)=\int\left|K_{p} d \sigma\right| \geqslant K[T]=8 \pi \cdot B[\gamma],
$$

where $\gamma$ is any core curve and $B[\gamma]$ its bridge index. For a knotted torus, equality is never attained:

$$
K(T)>8 \pi \cdot B[\gamma]
$$


Equality can occur only when $B[\gamma]=1$ which implies the torus is unknotted and

$$
K(T)=8 \pi .
$$

Recall that an immersed surface $M$ in $\mathbb{R}^{3}$ is tight in case $K(M)=\beta \cdot 2 \pi$, where $\beta=4-\chi(M)$ is the sum of the $\mathbb{Z}_{2}$-Betti numbers of the surface.

Before discussing the proof of Theorem 1, we first place the theorem in proper historical perspective. First, in 1976 Langevin and Rosenberg [7] proved that if $K(T)<16 \pi$, then the torus is unknotted. Later, Meeks [8], [9] and Morton [12] gave independent and different proofs that when the total curvature of an embedded surface $M_{g}$ of genus $g$ is less than $4 \pi(3+g)$, then the surface is isotopic to the standard embedding of the surface. An embedding $f: M \rightarrow \mathbb{R}^{3}$ of a closed surface $M$ is called isotopy tight when its total curvature is minimal in its isotopy class:

$$
K(f)=K[f] \text {. }
$$

The theorem of Meeks and Morton implies that a knotted surface $M_{g}$ in $\mathbb{R}^{3}$ with total curvature $4 \pi(3+g)$ must be isotopy tight. Kuiper and Meeks [6] proved that there do exist isotopy tight surfaces $M_{g}$ for every genus $g$ greater than two with $K\left(M_{g}\right)=4 \pi(3+g)$, but not for genus one or two. An important corollary from our statement of the generalized Fary-Fenchel-FoxMilnor theorem is that every isotopy tight torus is actually tight and therefore unknotted. It is still unknown if there exists an isotopy tight surface of genus two that is not tight. Theorem 2 is proved in steps:

After recalling known lemmas in $\S 1$, we show in $\S 2$ that a torus embedding $f: T \subset \mathbb{R}^{3}$ obeys the inequality $K[T] \leqslant 8 \pi B[\gamma]$. In $\$ 3$ we construct a core curve $\gamma$ so that $K[T] \geqslant 8 \pi B[\gamma]$, which proves $K[T]=8 \pi B[\gamma]$. In $\S 4$ we conclude by proving the inequality $K(T)>K[T]$ for a knotted torus. The appendix, $\$ 5$, gives the proof of a known theorem on knots, which we use in $\S 2$.

\section{An elementary property of the total curvature functional and a review of the fundamental lemma}

The total Gaussian curvature of a surface and the total classical curvature of a knot are related to another functional called the total curvature functional $\tau$, which makes sense for any smooth immersion of a closed $k$-manifold $M^{k}$ into $n$-dimensional euclidean space $\mathbb{R}^{n}$. Consider the $\operatorname{SO}(n)$-invariant measure on the set of all height functions in $\mathbb{R}^{n}$ that vanish at the origin $O \in \mathbb{R}^{n}$. The total curvature functiona! $\tau(F)$ of the smooth embedding $f: M^{k} \subset \mathbb{R}^{n}$ is defined to be the mean or expectation of the number of critical points $\mu(z \circ f)$ for all 
height functions $z: \mathbb{R}^{n} \rightarrow \mathbb{R}$, with respect to an $\mathrm{SO}(n)$-invariant measure. In the case of a knot $f: \gamma \subset \mathbb{R}^{3}$ or a closed surface embedding $f: M \subset \mathbb{R}^{3}$, the classical curvatures are related to the new ones as follows:

$$
K(\gamma)=\pi \cdot \tau(\gamma) \text { and } K(M)=2 \pi \cdot \tau(M)
$$

where by abuse of notation we write $\tau(\gamma)=\tau(f)$ and $\tau(M)=\tau(f)$ respectively. In terms of $\tau$ our formulas are simpler: In Theorem 1, for a knot $\gamma$ in $\mathbb{R}^{3}$

$$
\tau(\gamma) \geqslant \tau[\gamma]^{\cdot}=2 B[\gamma]
$$

with equality only for plane convex curves; and in Theorem 2, for a torus embedding $f: T \subset \mathbb{R}^{3}$,

$$
\tau(T) \geqslant \tau[T]=4 B[\gamma]
$$

with equality only for a tight torus, $\tau(T)=4$.

The following elementary lemma is straightforward to prove from the definition of $\tau$.

Lemma 1.1. If $M$ is a closed $k$-manifold embedded in $\mathbb{R}^{n}$ and $\partial N_{\varepsilon}(M)$ is the boundary of a regular $\varepsilon$-neighborhood of $M$ for some small positive $\varepsilon$, then

$$
\tau\left(\partial N_{\varepsilon}(M)\right)=2 \tau(M)
$$

In particular for a knot $\gamma$ in $\mathbb{R}^{3}$ :

$$
\tau\left(\partial N_{\varepsilon}(\gamma)\right)=2 \tau(\gamma)
$$

hence by $(0.3)$

$$
K\left(\partial N_{\varepsilon}(\gamma)\right)=4 K(\gamma)
$$

Suppose that $f: M \subset \mathbb{R}^{3}$ is an embedding of a closed surface in $\mathbb{R}^{3}$. If there exists a neighborhood $N(f)$ in the space of embeddings $f$ of $M$ in $\mathbb{R}^{3}$ in the $C^{1}$-norm such that for every affine transformation $A$ of $\mathbb{R}^{3}$ and for every $f^{\prime}$ in $N(f)$ the equation $\tau(f) \leqslant \tau\left(A f^{\prime}\right)$ is true, then $f$ is called relatively isotopy tight. It follows from this definition that every isotopy tight surface is relatively isotopy tight. The following theorem will be of the utmost importance in our later analysis of isotopy tight tori and its proof can be found in [6].

Fundamental Lemma 1.2. If $f: M \subset \mathbb{R}^{3}$ is a relatively isotopy tight embedding of a surface of genus $g$ and total Betti number $\beta=2+2 g$ in threedimensional euclidean space, then:

(A) There exists an integer $k$ and $k$ convex surfaces $\partial B_{1}, \cdots, \partial B_{k}$, such that the set $M_{K>0}$ of all points $p$ of $M$ with positive Gaussian curvature $K(p)>0$ is exactly the analogous set for $\bigcup_{i=1}^{k} \partial B_{i}$ :

$$
M_{K>0}=\bigcup_{i=1}^{k}\left(\partial B_{i}\right)_{K>0},
$$

and $\tau(F)=\beta+4(k-1)$. 
(B) The unique principal component $M_{i}^{+}$of $\partial B_{i} \cap M$ which contains $\left(\partial B_{i}\right)_{K>0}$ is obtained from $\partial B_{i}$ by deleting disjoint open plane convex discs in $\partial B_{i}$. We now reserve the word top circle of $M$ for the boundary of such a disc.

(C) The plane $\Pi(\gamma)$ of a top circle $\gamma$ supports some open neighborhood $U \subset M$ of $\Pi(\gamma) \cap M_{i}^{+}$; that is $U$ lies in one of the two closed half spaces bounded by $\Pi(\gamma)$. The set $\Pi(\gamma) \cap M_{i}^{+}$is then called a relative top set.

Conversely a surface $M \subset \mathbb{R}^{3}$ which satisfies properties (A), (B), (C) is relatively isotopy tight.

\section{An upper bound on the total curvature}

For any knot $\gamma$ in $\mathbb{R}^{3}$ and $\delta>0$ construct as follows two model tori $T_{\gamma}{ }^{ \pm}$in $\mathbb{R}^{3}$ with $\tau\left(T_{\gamma}^{ \pm}\right) \leqslant 4 B[\gamma]+\delta$.

Let $C$ be a smooth simple closed curve in the half-plane $\{(x, 0, z) \mid x \geqslant 0\}$ chosen so that the bounded component $Q$ of its complement is convex and so that $C \cap z$-axis $=\{(0,0, z)|| z \mid \leqslant 2\} \supset C \cap B_{1}$, where $B_{1}$ is the ball of radius 1. For small $\varepsilon>0$ let $C_{\varepsilon}$ be the curve at distance $\varepsilon$ from $C$ inside $Q$.

Definition. Given $\delta>0$, a knot $\gamma$ in $\mathbb{R}^{3}$ with bridge index $k$ is in $\delta$-position if

(a) $\gamma-B_{1}=C-B_{1}$,

(b) $z \mid \gamma$ is nondegenerate with $2 k$ critical points,

(c) $\tau(\gamma)<2 B[\gamma]+\delta / 2$.

Lemma 2.1. Given $\delta>0$, any knot in $\mathbb{R}^{3}$ is ambient isotopic to a knot in $\delta$-position.

Proof. Use the appendix (Lemma 5.1) to isotope $\gamma$ so that it satisfies (a) and (b). Then there is an isotopy of $\mathbb{R}^{3}$ which is the identity on $C-B_{1}$ and on $C \cap B_{1}$ is $(x, y, z) \rightarrow(x / t, y / t, z), 1 \leqslant t \leqslant \lambda$. Then for $\lambda$ large, after the isotopy,

$$
\tau(\gamma)<\tau[\gamma]+\delta / 2=2 B[\gamma]+\delta / 2
$$

For a knot $\gamma$ in $\mathbb{R}^{3}$ and $\delta>0$ let $T_{\gamma}^{+}$be the boundary of some small $\varepsilon$-tubular neighborhood of an isotope $\gamma_{\delta}$ of $\gamma$ in $\delta$-position. In particular, let $T_{0}^{+}$be an $\varepsilon$-tubular neighborhood of $C$. Then by Lemma 1.1, $\tau\left[T_{\gamma}^{+}\right] \leqslant \tau\left(T_{\gamma}^{+}\right)$ $=2 \tau(\gamma)<4 B[\gamma]+\delta$ and $\tau\left[T_{0}^{+}\right]=\tau\left(T_{0}^{+}\right)=4$.

Let $T_{0}^{-}$be the torus obtained by rotating $C_{\varepsilon}$ about the $z$-axis. Then, by construction, $\tau\left(T_{0}^{-}\right)=4$. In general, let $T_{\gamma}^{-}$be the smooth torus $\left(T_{\gamma}^{+} \cap B_{1}\right) \cup$ $\left(T_{0}^{-}-B_{1}\right)$.

For any height function $h$, the number of critical points $\mu\left(h \circ T_{\gamma}^{+}\right)-$ $\mu\left(h \circ T_{\gamma}^{-}\right)=\mu\left(h \circ T_{0}^{+}\right)-\mu\left(h \circ T_{0}^{-}\right)$so $\tau\left(T_{\gamma}^{+}\right)-\tau\left(T_{\gamma}^{-}\right)=\tau\left(T_{0}^{+}\right)-\tau\left(T_{0}^{-}\right)=0$. Therefore $\tau\left(T_{\gamma}^{-}\right)=\tau\left(T_{\gamma}^{+}\right)<4 B[\gamma]+\delta$. 
Lemma 2.2. For $\gamma$ a core curve of a torus $T$ in $\mathbb{R}^{3} \tau[T] \leqslant 4 B[\gamma]$.

Proof. $T$ is isotopic in $S^{3}$ to the boundary of a tubular neighborhood of $\gamma$. It follows that, for any $\delta>0, T$ is isotopic in $\mathbb{R}^{3}$ either to $T_{\gamma}^{+}$or $T_{\gamma}^{-}$, depending on whether the compactification point lies outside or inside the tubular neighborhood. Thus $\tau[T]<4 B[\gamma]+\delta$.

3. Construction of a core curve $\gamma$, and the proof of $\tau[T]=4 B[\gamma]^{1}$

Lemma 3.1. For a smooth torus embedding $f: T \subset \mathbb{R}^{3}$, let $z$ be a height function so that the restriction

$$
\varphi=z \circ f=z \mid T: T \rightarrow \mathbb{R}
$$

is nondegenerate with all critical points at different heights. Then there exists a smooth Jordan curve $\gamma$ on $T$, such that $\varphi \mid \gamma$ is nondegenerate with all critical points of $\varphi \mid \gamma$ contained in the set of index one critical points of $\varphi$, and if $p$ is such a critical point on $\gamma$, then neither of the two Jordan curves $\alpha$ and $\beta$ that make up the figure 8 level set component $W(p)$ of $\varphi$ containing $p$, bounds a disc on $T$. Also for any regular value $u$ of $\varphi$, and any component $V$ of $\varphi^{-1}(u)$, the Jordan curves $\gamma$ and $V$ intersect in one single point or not at all.

Remark. The same conclusion holds for any Morse function $\varphi: T \rightarrow \mathbb{R}$ with critical points at different levels.

Proof. The level set component $W(p)=\alpha \cup \beta$ of an index one critical point $p$ of the height function $\varphi$ is an immersed circle with one double point in the horizontal plane $z=z(p)$. It is the union of two embedded circles $\alpha$ and $\beta$ which meet in the common point $p$, where each has a corner. Evidently there is a small continuous deformation of $\beta$ inside $T$ near the saddle point $p$, by which $\beta$ is made disjoint from $\alpha$. By elementary surface topology of the torus we can distinguish three types of wedges $W(p)=\alpha \cup \beta$ in $T$ :

(a) Neither $\alpha$ nor $\beta$ bounds a disc in $T$; $\alpha$ and $\beta$, suitably oriented, are homologous and represent the same essential homology class in $H_{1}(T, \mathbb{Z})$.

(b) Exactly one among $\alpha$ and $\beta$, say $\beta$, bounds a disc in $T$ and then the other $\alpha$ represents an essential one-cycle $\{\alpha\} \neq 0$.

(c) Both $\alpha$ and $\beta$ bound discs: $\{\alpha\}=\{\beta\}=0$.

Let $l$ be the number of critical points of type (a), $m$ the number of type (a) or (b), and $n$ the total number of critical points of index one, $l \leqslant m \leqslant n$. Any two nonbounding circles obtained as above for index one critical points $p_{i}$ and

\footnotetext{
${ }^{1}$ Morton [12] already gave a proof leading to $4 B[\gamma] \leqslant \tau(T)$ for $T^{\text {int }}$ a solid torus. However, he argues by contradiction and does not actually construct the core curve $\gamma$.
} 
$p_{j} \neq p_{i}$ are at different levels and disjoint in $T$, so all are embedded circles in one and the same homology class in $H_{1}(T, \mathbb{Z})$.

If $W(p)$ is of type (b), then by elementary surface topology, $T \backslash W(p)$ has two components, one (by definition of type (b)) an open disc with boundary $\beta$, and the other an open annulus with $\alpha$ as limit at one end and $W(p)=\alpha \cup \beta$ as limit set at the other end. These ends of the annulus approach $W(p)$ from different sides of the plane $z=z(p)$.

If $W(p)$ is of type (a) then again $T \backslash W(p)$ has two components, one an open disc having $W(p)$ as limit set, and the other an open annulus having $\alpha$ as limit set at one end and $\beta$ as limit set at the other end. The ends of the annulus approach $W(p)$ from the same side of the plane $z=z(p)$.

Let $p_{1}, \cdots, p_{m}$ be the $m \geqslant 2$ index one critical points of type (a) or (b), with associated level set wedges $W\left(p_{i}\right), \cdots, W\left(p_{m}\right)$. Then there are $2 m \geqslant 4$ components in

$$
T \backslash \bigcup_{i=1}^{m} W\left(p_{i}\right)
$$

Each component is either an open annulus without critical points for $\varphi$, with limit set contained in two distinct level sets $W\left(p_{i}\right)$ and $W\left(p_{j}\right), j \neq i$, or it is an open disc with limit set contained in one single level set $W\left(p_{i}\right)$ of $\varphi$. Observe that any such annulus is transversal to every horizontal plane between the ends and any horizontal section of each open annulus represents an essential homology class on $T$, and as these sections are mutually disjoint they are homologous in $H_{1}(T ; \mathbb{Z})$ if suitably oriented.

At each end of each annulus there is a critical point, so that we can make a sequence that closes, consisting of closures of annuli $A_{1}, A_{2}, \cdots, A_{r}$ and relevant critical points $p_{1}, \cdots, p_{r}$, for which

$$
p_{i} \in A_{i} \cap A_{i+1}, \quad p_{i}=p_{i+r}, A_{i}=A_{i+r} .
$$

The union of these annuli together with the open discs, one adjacent to each among $p_{1}, \cdots, p_{r}$, yields a closed surface which is part of $T$. This must be all of $T$, and therefore $r=m$.

Since $\varphi \mid \operatorname{Int}\left(A_{i}\right)$ has no critical points, there exist smooth Jordan arcs $\gamma_{i} \subset A_{i}$ satisfying:

(1) $\gamma_{i} \cap \partial A_{i}=\left\{p_{i-1}, p_{i}\right\}, i \bmod m$

(2) $\tilde{\gamma}=\bigcup_{i=1}^{m} \gamma_{i}$ is a piecewise smooth Jordan curve, smooth at points of type (a), and

(3) $\varphi \mid \tilde{\gamma}$ has critical points precisely at $\left\{p_{1}, \cdots, p_{m}\right\}$.

If $\alpha_{i}$ does not bound a disc in $T$ but $\beta_{i}$ does bound a disc in $T$, then the function $\varphi \mid \tilde{\gamma}$ is strictly increasing or decreasing in a neighborhood of the point $p_{i}$. Thus, after a small perturbation of $\tilde{\gamma}$ at each such critical point $p_{i}$ of $f$, we 
may assume that the perturbed curve $\gamma$ is smooth and has no longer a critical point near $p_{i}$. It is straightforward to check that $\gamma$ satisfies the remaining properties described in Lemma 3.1, which completes the proof of the lemma.

Observe that $\varphi \mid \gamma$ has $2 k=l$ critical points, where $l$ is the (even) number of index one critical points of type (a) of $\varphi$ on $T$.

Lemma 3.2. If $T$ is an embedded torus in $\mathbb{R}^{3}$, with total curvature $\tau(T)$, then there exists a core curve $\gamma$ for which $4 B[\gamma] \leqslant \tau(T)$.

Proof. By definition of $\tau$ as an average there exists a height function $z: \mathbb{R}^{3} \rightarrow \mathbb{R}$, such that $\varphi=z \mid T$ is nondegenerate with the smallest number, say $2 n$, critical points and at different heights. The number of index one critical points is then $n$ and $2 n \leqslant \tau(T)$.

In Lemma 3.1 we constructed a Jordan curve on $T$, which curve we now call $\bar{\gamma}$, for which $\varphi \mid \bar{\gamma}$ is nondegenerate with $2 k=l \leqslant m \leqslant n$ critical points. Then

$$
4 B[\bar{\gamma}] \leqslant 4 k \leqslant 2 n \leqslant \tau(T) .
$$

It remains to construct a core curve $\gamma$ isotopic to $\bar{\gamma}$, so that $B[\gamma]=B[\bar{\gamma}]$.

The required $\gamma$ will be a copy of $\bar{\gamma}$, inside the closure of the standard component $T^{u}$ of $S^{3} \backslash T$.

Let $\varphi \mid \bar{\gamma}$ attain its minimal value $\lambda$ at the critical point $p, \varphi(p)=\lambda$. For $\delta>0$ sufficiently small we can assume by Lemma 3.1 .

(1) $\varphi^{-1}((\lambda, \eta]), \eta=\lambda+\delta$, contains no critical point of $\varphi$,

(2) the smooth 1-manifold $\varphi^{-1}(\eta)$ contains components $\zeta_{1}, \zeta_{2}$, Jordan curves near to $p$, and these are the only components of $\varphi^{-1}(\eta)$ which do not bound discs on $T$.

Let $F=z^{-1}(\eta)$ be the plane at height $z=\eta$ and let $\zeta_{3}, \cdots, \zeta_{r}$ be the other components of $P \cap T$. Each of them bounds a disc in $T$. Suppose $\zeta_{j}, j \geqslant 3$, is an innermost circle among $\zeta_{1}, \cdots, \zeta_{r}$ in $P$. It bounds a disc $D_{1}$ in $P$ and a disc $D_{2}$ in $T$. Then $D_{1} \cup D_{2}$ is an embedded two-sphere and one of the two open three-balls components of $S^{3} \backslash\left(D_{1} \cup D_{2}\right), S^{3}=\mathbb{R}^{3} \cup \infty$, has no point in common with $T$. So $D_{2}$ in $T$ can be ambient isotoped in $S^{3}$ over that component and after a slight final perturbation we have annihilated $\zeta_{j}$ from $P \cap T$. Only some small neighborhood of $D_{2}$ in $T$ is involved in this ambient isotopy of $T$. In particular $\bigcup_{i=1}^{m} A_{i}$ and $\bar{\gamma}$ are untouched by this isotopy of $T$, where $A_{i}$ are the annuli defined in the proof of Lemma 3.1. Proceeding in the same way inductively, we reach a situation where either $\zeta_{1}$ or $\zeta_{2}$, say $\zeta_{1}$, is innermost and it bounds an open disc $D$ in $P$ disjoint from (the new image of) $T$. By Alexander's theorem [13] the component of $S^{3} \backslash T$ which contains the interior of $D$ is a standard component $T^{u}$. Since $\gamma$ intersects precisely in one point an essential curve in $T$ bounding a disk in $T^{u}, \bar{\gamma}$ is isotopic to a core 
curve $\gamma$ of $T$. Then

$$
4 B[\gamma] \leqslant 4 k \leqslant 2 n \leqslant \tau(T)
$$

which completes the proof of the lemma.

Since $4 B[\gamma] \leqslant \tau\left(T^{\prime}\right)$ holds for any $T^{\prime}$ isotopic to $T$, Lemma 2.1 implies the corollary: $\tau[T]=4 B[\gamma]$.

\section{Isotopy-tight knotted torus}

Theorem 2b. There is no isotopy-tight knotted torus in $\mathbb{R}^{3}$.

Proof. Let $f: T \subset \mathbb{R}^{3}$ be a knotted isotopy tight torus with total curvature

$$
\tau(T)=\tau[T]=4 B[\gamma]=4 k .
$$

We will deduce a contradiction.

If $z \mid T$ is a nondegenerate height function, then it has exactly $4 k$ critical points of which $2 k$ are of index one, which we can assume to have different heights. By the construction in $\S 3$ of the core curve $\gamma$ there must be at least $2 B[\gamma]=2 k$ index one critical points of type (a). Hence all are of type (a) and their wedges $W_{i}=\alpha_{i} \vee \beta_{i}, i=1, \cdots, k$, have nonbounding components $\alpha_{i}$ and $\beta_{i}$ in $T$. Recall Lemma 1.2, where $k$ convex surfaces $\partial B_{i}$ are constructed with principal compact parts of $M=T: M_{i}^{+}=T_{i}^{+} \subset \partial B_{i}, i=1, \cdots, k$, obtained by deleting from each $\partial B_{i}$ open convex plane discs. The remaining part of $T$, $T \backslash \bigcup_{i=1}^{k} T_{i}^{+}$, has open components, say $T_{j}^{-}, j=1, \cdots, r$, whose ends have a union of one or more plane convex top-circles as limit sets.

The Gauss-curvatures on $T_{i}^{+}$and $T_{j}^{-}$are $K \geqslant 0$ and, $K \leqslant 0$, respectively. Suppose $T_{i}^{+}$has only one boundary component, the top circle $\zeta$. Assume $\zeta$ lies in the horizontal plane $z=0$ which then supports $T_{i}^{+}$. If $z^{\prime}$ is a height function very near to $z$ and $z^{\prime} \mid T$ is nondegenerate, then it has near to $\zeta$ a critical point of index one with associated wedge $W=\alpha \cup \beta$. This wedge is contained in some small neighborhood in $T$ of the disc $T_{i}^{+}$. So $\alpha$ and $\beta$ are both bounding in $T$ which is a contradiction. Therefore, $T_{i}^{+}$is obtained from $\partial B_{i}$ by deleting at least two open plane convex discs, and the Euler characteristic is

$$
\chi\left(T_{i}^{+}\right) \leqslant 0, \quad i=1, \cdots, k .
$$

A component $T_{j}^{-}$cannot have only one end with limit set a top-circle either, because the distance to the plane of that top-circle would have to have a positive maximum on the open disc $T_{j}^{-}$, for which points of positive curvature $K>0$ are needed in $T_{j}^{-}$. So also $\chi\left(T_{j}^{-}\right) \leqslant 0$. It might be that on $\partial B_{i}$ two open discs deleted are in the same plane and even tangent to each other, so that $T_{i}^{+}$ 
would not be a two-dimensional surface with boundary. But then we can enlarge the parts $T_{i}^{+}$a little bit by adding small collars from $\bigcup_{j=1}^{r} T_{j}^{-}$to obtain a surface with boundary. It is easily seen that under all circumstances there is the sum-formula:

$$
0=\chi(T)=\sum_{i=1}^{k} \chi\left(T_{i}^{+}\right)+\sum_{j=1}^{r}: \chi\left(S T_{j}^{-}\right) .
$$

As $\chi\left(T_{i}^{+}\right) \leqslant 0$ and $\chi\left(T_{j}^{-}\right) \leqslant 0$, it follows that

$$
\chi\left(T_{i}^{+}\right)=0, \quad i=1, \cdots, k, \quad \text { and } \quad \chi\left(T_{j}^{-}\right)=0, \quad j=1, \cdots, r .
$$

We conclude that for all $i$ and $j, T_{i}^{+}$is obtained from $\partial B_{i}$ by deleting exactly two open plane convex discs, and $T_{j}^{-}$is an open annulus. Then we obtain a cyclically ordered covering of $T$ by alternating pieces $T_{i}^{+}$and open annuli $T_{j}^{-}$:

$$
T=T_{1}^{+} \cup T_{2}^{-} \cup T_{2}^{-} \cup \cdots \cup T_{k}^{+} \cup T_{k}^{-},
$$

where

$$
\left.\begin{array}{l}
\partial T_{i}^{+}=\xi_{i} \cup \eta_{i} \\
\partial \bar{T}_{i}^{-}=\eta_{i} \cup \xi_{i+1}
\end{array}\right\} i \bmod k .
$$

$\bar{T}_{i}^{-}$is the closure of $T_{i}^{-}$in $T, \xi_{1}, \eta_{1}, \xi_{2}, \eta_{2} \cdots$ are successive essential top-circles on $T$, and all such are counted.

For any set $X \subset \mathbb{R}^{3}$ denote by $\mathscr{H}(X)$ the smallest convex set containing $X$, the convex hull of $X$. A variant of Lemma 5.1 in [6] for our case is

Lemma 4.1. $T_{i}^{+} \cap \mathscr{H}\left(\xi_{i+1}\right)=\varnothing$, and $T_{i+1}^{+} \cap \mathscr{H}\left(\eta_{i}\right)=\varnothing, i \bmod k$.

Proof. Let the top-circle $\xi_{i+1}$ be in the horizontal plane $\Pi\left(\xi_{i+1}\right)$ with equation $z=0$ and $T_{i+1}^{+}$under the plane. Suppose $T_{i}^{+}$meets the plane disc $\mathscr{H}\left(\sigma \mathrm{x}_{i+1}\right)$, hence in its interior (see Figure 1(a)). We fill the ends of the annulus $T_{i}^{-}$with the convex disc $\mathscr{H}\left(\xi_{i+1}\right)$ and $\mathscr{H}\left(\eta_{i}\right)$ to obtain a piecewise smooth immersed two-sphere

$$
S^{2}=T_{i}^{-} \cup \mathscr{H}\left(\xi_{i+1}\right) \cup \mathscr{H}\left(\eta_{i}\right) \subset \mathbb{R}^{3} .
$$

Since $T_{i}^{-}$has nonpositive curvature, we see that the sphere $S^{2}$ lies in the convex body $\mathscr{H}\left(\xi_{i+1} \cup \eta_{i}\right)$.

Take points $p$ and $q$ in the interior of $T_{i}^{+} \subset \partial B_{i}$, near to maximal and minimal height points of $z$ on $T_{i}^{+}$respectively. Connect $p$ and $q$ by one arc $\sigma_{1}$ inside $T_{i}^{+}$, and one arc $\sigma_{2}$ outside $\mathscr{H}\left(T_{i}^{+} \cup \xi_{i+1} \cup \eta_{i}\right)$, starting at $p$ going vertically up and arriving at $q$ vertically up. The arcs $\sigma_{1}$ and $\sigma_{2}$ can be chosen such that $\sigma_{1} \cup \sigma_{2}$ is an embedded circle $\sigma$, which meets the immersed two-sphere $S^{2}$ only in one point of the plane disc $\mathscr{H}\left(\xi_{i+1}\right)$ and the intersection is transversal. This is impossible. The same argument gives the second relation. 
Lemma 4.2. If $\xi_{i+1}$ has a point $x$ in $\mathscr{H}\left(T_{i}^{+}\right)$, then

$$
\eta_{i} \cap \mathscr{H}\left(T_{i+1}^{+}\right)=\varnothing .
$$

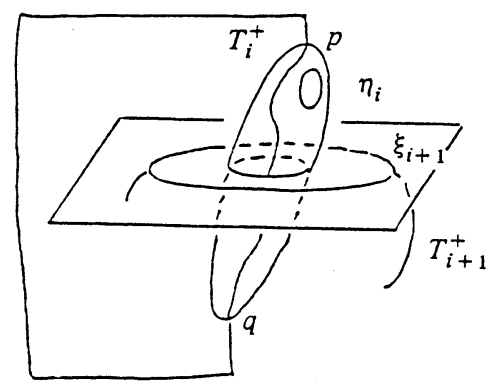

a)

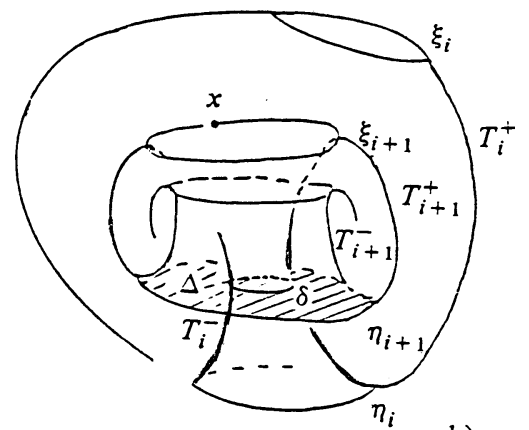

b)

FIGURE 1

Proof. See Figure 1(b). As $x \in \xi_{i+1} \subset T_{i+1}^{+}$is a point in $\mathscr{H}\left(T_{i}^{+}\right)$we see that $T_{i+1}^{+}$has a point in $\mathscr{H}\left(T_{i}^{+}\right)$. By Lemma 4.1 we know

$$
T_{i+1}^{+} \cap\left[T_{i}^{+} \cup \mathscr{H}\left(\eta_{i}\right)\right]=\varnothing,
$$

so the convex surface $\partial B_{i+1}$ can get out of $\mathscr{H}\left(T_{i}^{+}\right)$only through the disc $\mathscr{H}\left(\xi_{i}\right)$. But then $B_{i+1}=\mathscr{H}\left(T_{i+1}^{+}\right)$cannot meet $\mathscr{H}\left(\eta_{i}\right)$, nor $\eta_{i}$, and the lemma follows.

If $\xi_{i+1}$ has no point in $\mathscr{H}\left(T_{i}^{+}\right)$, then

$$
\xi_{i+1} \cap \mathscr{H}\left(T_{i}^{+}\right)=\varnothing .
$$

So we can assume either (i) or (ii). We will assume (i).

Lemma 4.3. If $\eta_{i} \cap \mathscr{H}\left(T_{i+1}^{+}\right)=\varnothing$, then

$$
\mathscr{H}\left(T_{i+2}^{+}\right) \subset \mathscr{H}\left(T_{i+1}^{+}\right) \text {and } \eta_{i+1} \cap \mathscr{H}\left(T_{i+2}^{+}\right)=\varnothing .
$$

Proof. See Figure 1(b). Choose a height function $z$ so that the disk $\mathscr{H}\left(\eta_{i+1}\right)$ is horizontal and in the plane $z=0$. If $\eta_{i} \cap \mathscr{H}\left(T_{i+1}^{+}\right)=\varnothing$, then the horizontal disk $\mathscr{H}\left(\eta_{i+1}\right)$ separates the ends $\eta_{i}$ and $\xi_{i+1}$ of $T_{i}^{-}$from each other, and $T_{i}^{-}$meets the convex ball

$$
B_{t}=\mathscr{H}\left(T_{i+1}^{-}\right) \cap(z \geqslant t)
$$

for almost all small $t \geqslant 0$ in a smooth 2-manifold with smooth boundary, which must be an annulus because $z$ cannot create handles (no maxima or minima) on (the interior of) $T_{i+1}^{-}$. With a small vertical ambient isotopy we can therefore assume $\delta$ itself to be one smooth circle (in fact one can prove that $\delta$ must be a smooth circle from the beginning). The annulus $A \subset T_{i}^{-}$between 
$\xi_{i+1}$ and $\delta$ divides $\mathscr{H}\left(T_{i+1}^{+}\right)$in two parts. Let $W$ be the closure of the part that contains $\eta_{i+1}$. Its boundary is $\partial W=T_{i+1}^{+} \cup A \cup \Delta$, where $\Delta$ is the plane annulus between $\delta$ and $\eta_{i+1}$.

The highest point of $\bar{T}_{i+1}^{-}$lies inside $W$, as well as on the top circle $\xi_{i+2}$, hence also on $T_{i+2}^{+}$. The convex body $B_{i+2}=\mathscr{H}\left(T_{i+2}^{+}\right)$as well as $T_{i+2}^{+}$therefore has a point inside $W$, and $T_{i+2}^{+}$cannot escape through $\partial W$ except through $\Delta \subset \mathscr{H}\left(\eta_{i+1}\right)$. But by Lemma 4.1 this is excluded also, as $T_{i+2}^{+} \cap \mathscr{H}\left(\eta_{i+1}\right)=\varnothing$. Therefore $T_{i+2}^{+} \subset W \subset \mathscr{H}\left(T_{i+1}^{+}\right)$, and so $\mathscr{H} T_{i+2}^{+} \subset \mathscr{H} W \subset \mathscr{H}\left(T_{i+1}^{+}\right)$as well as $\eta_{i+1} \cap \mathscr{H}\left(T_{i+2}^{+}\right)=\varnothing$, and Lemma 4.3 is proved.

Conclusion. By Lemma 4.2 we can assume $\eta_{i} \cap \mathscr{H}\left(T_{i+1}^{+}\right)=\varnothing$ so that the conclusion of Lemma 4.3 holds for our case. Then we can apply Lemma 4.3 inductively starting with index $i=1$ for $i=1,2,3, \cdots, k, k+1=1 \bmod k$ and get the impossible sequence of proper inclusions

$$
\mathscr{H}\left(T_{1}^{+}\right) \subset \mathscr{H}\left(T_{2}^{+}\right) \subset \cdots \subset \mathscr{H}\left(T_{k}^{+}\right) \subset \mathscr{H}\left(T_{1}^{+}\right) .
$$

This proves Theorem $2 b$.

\section{Appendix. A lemma on knots}

Lemma 5.1. If $\gamma$ is a knot in $\mathbb{R}^{3}$ with bridge index $k$, then there exists a knot $\alpha$ isotopic to $\gamma$ which satisfies:

(1) $z \mid \alpha$ is a Morse function with $2 k$ critical points.

(2) $\alpha \cap B_{1}=\left\{p \in \mathbb{R}^{3}:|p| \leqslant 1\right\}$.

(3) $\alpha \cap \partial B_{1}=\{(0,0,1),(0,0,-1)\}$.

(4) There is a closed interval $I \subset \alpha$ with $\partial I=\alpha \cap \partial B_{1}$ and $z \mid I$ has no interior critical points.

(5) I lies in the vertical half disc

$$
P=\left\{(x, y, z) \in B_{1}: y=0, x \geqslant 0\right\} .
$$

(6) The component $C$ of $P \backslash I$ that contains $(1,0,0)$ is disjoint from $\alpha$.

Remark. Observe that Lemma 2.1 is an immediate consequence of Lemma 5.1 ; just apply a suitable ambient isotopy to $\alpha$ as obtained in Lemma 5.1 under which any point $q$ moves in its horizontal plane $z=z(q)$.

Proof. The existence of a knot $\alpha_{1}$ satisfying all of the properties except (6) is clear. Choose the knot $\alpha_{1}$ to satisfy not only properties (1)-(5), but suppose also that $\alpha_{1}$ is transverse to $C$, and that the intersection points of $C \cap \alpha_{1}$ are not critical for $z \mid \alpha_{1}$ and have different $z$-values.

Choose one of these points $p$ and for convenience let $z(p)=0$. Take a small closed interval neighborhood $J$ of $p$ on $\alpha$ such that $z \mid J$ is nondegenerate (monotone) and such that

$$
V=\left\{(x, y, z) \in B_{1}: \exists(t, y, z) \in J \text { and } x \geqslant t\right\}
$$


is an embedded closed disc disjoint from $\alpha_{1} \backslash J$ (see Figure 2). The piecewise smooth knot

is $C^{0}$-isotopic to $\alpha_{1}$.

$$
\alpha_{2}=\left(\alpha_{1} \cup \partial V\right) \backslash \operatorname{Int} J
$$

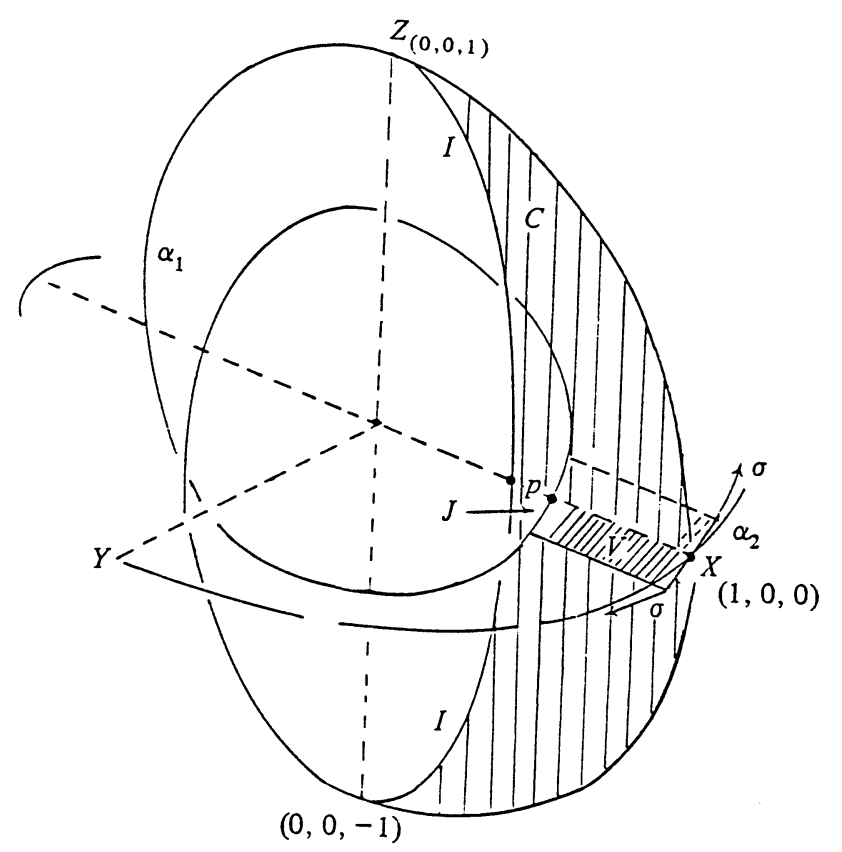

FIGURE 2

Let $\sigma$ be a smooth arc in $\partial B_{1}$ that connects the ends of the arc $\partial V \cap \partial B_{1}$ such that $z \mid \sigma$ has no interior critical points and $\sigma \cap C=\varnothing$. The piecewise smooth knot

$$
\alpha_{3}=\left(\alpha_{2} \cup \sigma\right) \backslash \operatorname{Int}\left(\partial V \cap \partial B_{1}\right)
$$

is $C^{0}$-isotopic to $\alpha_{2}, \alpha_{1}$, and $\gamma$. After a small perturbation we obtain a smooth knot $\alpha_{4}$ which is isotopic to $\gamma$, satisfies properties (1)-(5), and has one less intersection point with $C$ than $\alpha_{1}$. After a finite number of such isotopies, one for each point of $C \cap \alpha_{1}$, we find the required knot $\alpha$ for which (1)-(6) hold.

\section{References}

[1] R. H. Crowell \& R. H. Fox, Introduction to knot theory, Ginn and Company, Boston, 1963.

[2] I. Fary, Sur la courbure totale d' une courbe gauche faisant un noeud, Bull. Soc. Math. France 77 (1949) 128-138.

[3] W. Fenchel, Uber die Krümmung und Windung geschlossener Raumkurven, Math. Ann. 101 (1929) 228-252. 
[4] R. H. Fox, On the total curvature of some tame knots, Ann. of Math. (2) 52 (1950) 258-261.

[5] N. H. Kuiper, Convex immersions of closed surfaces in $E^{3}$, Comment Math. Helv. 35 (1961) 85-92.

[6] N. H. Kuiper \& W. H. Meeks III, Total curvature of knotted surfaces, Invent. Math. 77 (1984) 25-69.

[7] R. Langevin \& H. Rosenberg, On the curvature integrals and knots, Topology 15 (1976) 405-416.

[8] W. H. Meeks III, Lectures on Plateau's problem, IMPA, Rio de Janeiro, Brazil, 1978.

[9] - The topological uniqueness of minimal surfaces in three dimensional euclidean space, Topology 20 (1981) 389-411.

[10] J. W. Milnor, On the total curvature of knots, Ann. of Math. (2) 52 (1950) 248-257.

[11] On the total curvature of closed space curves, Math. Scand. 1 (1953) 289-296.

[12] H. R. Morton, A criterion for an embedded surface to be knotted, Lecture Notes in Math., Vol. 722, Springer, Berlin, 1977, 92-98.

[13] D. Rolfsen, Knots and links, Math. Lecture Series, Vol. 7, Publish or Perish, Berkeley, 1976.

[14] H. Schubert, Uber eine numerische Knoteninvariante, Math. Z. 61 (1954) 245-288.

\section{Institut des Hautes Études SCIENTIFiques RICE UNIVERSITY}

